\title{
A Simple Method for Photoconductivity Measurement in Lithium Niobate ${ }^{\dagger}$
}

\author{
Marco Bazzan 1,*(D), Laurent Guilbert ${ }^{2}$ and Michel Aillerie ${ }^{2}$ (D) \\ 1 Dipartimento di Fisica e Astronomia, Università di Padova, Via Marzolo 8, 35131 Padova, Italy \\ 2 CentraleSupélec, Université de Lorraine, LMOPS, F-57000 Metz, France; \\ laurent.guilbert@univ-lorraine.fr (L.G.); aillerie@metz.supelec.fr (M.A.) \\ * Correspondence: marco.bazzan@unipd.it \\ + We dedicate this article to the honorable memory of our colleague and friend Professor \\ Nikiforos Theofanous.
}

Received: 30 April 2020; Accepted: 22 May 2020; Published: 1 June 2020

\begin{abstract}
A simple and effective technique to characterize the photoconductivity (PC) of lithium niobate is presented. The technique is based on the modulation of the external field and on the observation of the optical response of the material as a function of the intensity of a gaussian beam using a Tardy's polarimetric setup in the $r_{22}$ configuration. When the temporal period of the modulation is larger than the Maxwell time of the material, the effect of the PC can be detected observing the kinetics of the screening effect of the external applied field. This approach allows measuring a wide dynamic range up to high light intensities with good accuracy using a standard oscilloscope and with no need for charge collection electrodes. The technique is demonstrated by comparing two samples, the first possessing a standard congruent composition, the second being doped with $\mathrm{Zn}$ in order to boost the PC.
\end{abstract}

Keywords: photoconductivity; experimental techniques; lithium niobate

\section{Introduction}

Lithium niobate (LN) plays a key role in modern photonics for the realization of electro-optic phase modulation systems or in nonlinear optical devices. However, this material is plagued by the so-called photorefractive (PR) damage, which is one of the main factors limiting its use at high light intensities such as those encountered in waveguides or in nonlinear optical applications [1,2]. The PR damage results in the perturbation of the material refractive index inside the illuminated area. It is caused by photo-excitation and migration of electric charges inside the material bulk when this is illuminated with strong nonuniform light. This charge redistribution gives rise to an intense electric field that locally modifies the material refractive index via the electro-optic effect [2]. In an effort to eliminate or at least mitigate this problem, it has been found that doping a given material with suitable elements, the so-called optical damage resistant impurities (ODRI), may largely modify its PR sensitivity [3]. While a detailed microscopic modeling of this effect requires a considerable effort [4-7], the macroscopic reason for this improvement is generally related to a marked increase in the material photoconductivity (PC) preventing the buildup of strong internal fields [8]. Therefore, a direct measure of the PC allows in principle assessing the resistance to PR damage in a batch of similar samples, as well as being an important parameter by itself. To our knowledge the routine methods to measure the photoconductivity generally can be traced back either to direct measurements with an electro-meter while the sample is uniformly illuminated, either to indirect ones where the time-evolution of photorefractive effects is measured and related to PC. The first kind of techniques requires to measure impedances that, in a bulk sample, are typically higher than hundreds of gigaohms 
not to mention the fact that to attain the same power density levels obtained when the beam is focused to a small diameter as it is the case in typical applications it would be necessary to use a high power laser beam to illuminate uniformly the sample.

For this reason, indirect optical methods exploiting PR phenomena are generally preferred. Several solutions have been provided, ranging from the simple observation of the beam defocusing as a consequence of the optical damage either isotropically [9] or anisotropically [10], to the recording/erasing dynamic of photorefractive gratings [11-15]. However, both beam defocusing and holographic recording require a sophisticated modeling to obtain a robust estimate of the kinetics, especially when Gaussian beams are used, see, for example, [15-17]. Other approaches may be based on the recording kinetics of phase images [7], again taking advantage of the photorefractive effect. In this case it is required a time-resolved analysis of the phase image formation, which is feasible but again brings with it a certain degree of technical complication.

In this paper we report on a simple method to measure PC in LN, provided that the electric-fieldinduced refractive index change can be continuously measured in some way. In our case, we exploited a Tardy's polarimetric setup to visualize the electro-optic index change with the sample in the so-called $r_{22}$ configuration which consists in having the beam propagating parallel to the optical axis and the external field applied perpendicular to it. In particular, this configuration allows to limit the problems related to the photo-galvanic effect which, in other configurations (e.g., with the beam propagating perpendicular to the optical axis), would produce an additional internal field perturbing the result of the measurement. By proper adaptation, the same measuring principle may be extended to other materials and configurations. Using this technique we measured the PC in a congruent undoped sample and in a Zn-doped one with increased resistance to PR damage.

\section{Method}

The basic idea of the method is to apply an external field to the sample and use the electro-optic induced birefringence as a probe to measure the decay of the applied field due to the PC.

A simplified scheme of our setup is shown in Figure 1. An Ar laser $(\lambda=514 \mathrm{~nm})$ is attenuated to a selected intensity using the combination of a half waveplate and a polarizing beamsplitter. The exiting beam is converted to circular polarization by a quarter waveplate and focused to a waist of about $83 \mu \mathrm{m}$ using a lens. The resulting confocal parameter of about $8 \mathrm{~cm}$ is safely longer than the length of our sample (typically not longer than $3 \mathrm{~cm}$ ), so that we can consider that the beam does not change its size inside the sample. The latter is a parallelepiped with length $L$ along the beam direction and thickness $d$. On two faces parallel to the beam, two electrodes are painted and connected to a high voltage generator driven by a square wave signal that applies along the whole sample length a voltage $V$ of alternating sign. One of the crucial aspects of our method is the use of the so-called $r_{22}$ configuration, in which the beam propagates parallel to the optical axis with circular polarization and the field is applied along the direction lying in one of the three mirror planes and perpendicular to the optical axis . Following standard notation for LN, the former direction will be labeled as z-axis and the latter as $y$-axis. A first advantage of this configuration is that at zero field, the sample is not birefringent so that the signal is much more stable and immune from external perturbations, such as temperature fluctuations and so on. Second, if the incident beam is circularly polarized, it is possible to minimize the impact of photo-galvanic internal fields that would develop in LN if other configurations or other polarization states would be used. This occurrence results from an analysis of the photogalvanic and electro-optic tensors and is detailed in the Supplementary Materials. In this way the measured electro-optic changes can be related exclusively to the external field. 


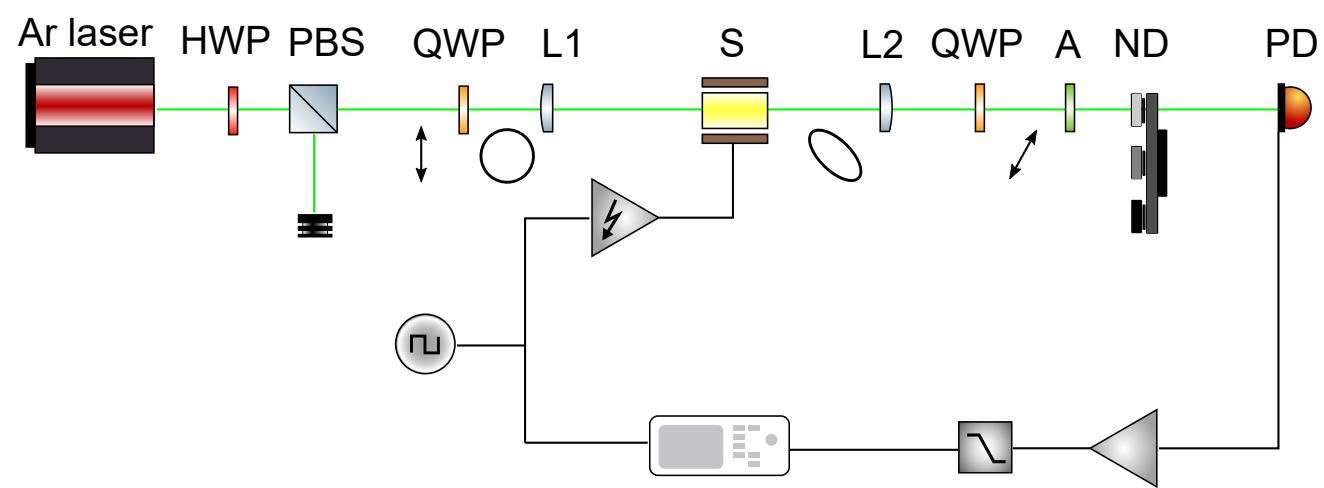

Figure 1. Simplified scheme of the setup. The elements composing it are: half waveplate (HWP); polarizing beamsplitter (PBS); quarter wave plate (QWP); lens $f=250 \mathrm{~mm}$ (L1); sample (S); lens $f=60 \mathrm{~mm}$ (L2); analyzer (A); neutral density filter wheel (ND); photodiode (PD). The sample is placed inside the Rayleigh range of the beam created by the lens L1, while the lens L2 images the output face of the sample on the photodiode. The arrows, circle and ellipse indicate the polarization state of the beam along the Tardy's setup.

The electro-optic induced birefringence is measured using a Tardy's setup, a variant of the de Sénarmont arrangement [18], where the incident light is circularly polarized before entering the sample. Let us assume, to begin with, that a static uniform electric field is present inside the sample along the $y$ - direction. In our configuration, the sample is oriented with the optical axis parallel to the beam propagation direction so that when the applied field is zero, the beam sees ideally no birefringence and the beam polarization before and after the sample remains circular. However, when an electric field is applied along the $y$-direction, the electro-optic effect induces a birefringence:

$$
\Delta n=n_{x}-n_{y}=n_{o}^{3} r_{22} E_{2}=n_{o}^{3} r_{22} V / d
$$

where $E_{2}$ is the electric field component in the $y$ - direction, $n_{0}=2.333$ is the lithium niobate ordinary refractive index at $\lambda=514 \mathrm{~nm}$ and $r_{22}=6.8 \frac{\mathrm{pm}}{\mathrm{V}}$ is the pertinent element of the electro-optic tensor [19]. Because of the induced birefringence, a phase shift $\Delta \phi=2 \pi L \Delta n / \lambda$ is accumulated between the two polarization components along $x$ - and along $y$-. By a proper adjustment of the analyzer angle, and for not too high values of the applied fields so that $\Delta \phi \ll \pi$, the Tardy's setup can be operated at the so-called linear point [20]. Thus, the PD signal is to a good approximation proportional to the field inside the sample:

$$
V_{P D}=A E_{2}+B
$$

where $A$ and $B$ are constants depending on the particular choice of experimental parameters like beam intensity, sample length, attenuators setting and so on.

Now, if the sample is photoconductive, the field inside the illuminated region slowly decays to zero because of the screening effect of the photoexcited free charges. For not too strong light intensities $I$, the PC can be assumed proportional to the light intensity itself:

$$
\sigma=\sigma_{0} I
$$

If the beam spot was illuminated by a flat-top beam with uniform intensity, the response to a voltage step would be a mono-exponential decay with a Maxwell time constant:

$$
\tau=\frac{\varepsilon \varepsilon_{0}}{\sigma}=\frac{\varepsilon \varepsilon_{0}}{\sigma_{0} I}
$$


where $\varepsilon_{0}$ is the vacuum permittivity and $\varepsilon=44$ is the relative dielectric constant of lithium niobate in the $x y$ plane, i.e., perpendicular to the propagation direction. However, for a Gaussian beam the intensity is not uniform so that different parts of the illuminated region decay with different time constants. If the sample length is shorter than the confocal parameter of the beam, we may neglect the dependence of the illumination profile along the propagation distance and the time constant can be assumed to be position-dependent with an expression of this kind:

$$
\tau(r)=\frac{\varepsilon \varepsilon_{0}}{\sigma_{0} I_{0}} \exp \left(\frac{r^{2}}{2 w^{2}}\right)=\tau_{0} \exp \left(\frac{r^{2}}{2 w^{2}}\right)
$$

where $\tau_{0}=\frac{\varepsilon \varepsilon_{0}}{\sigma_{0} I_{0}}$ is the response time for the peak illumination intensity $I_{0}$ and $r$ is the radial coordinate. The signal measured by the photodiode comes from the integration of the different parts of the beam evolving with the appropriate time constant, which results in the simple expression:

$$
V_{P D}=A \frac{\left[1-\exp \left(-\frac{t}{\tau_{0}}\right)\right]}{\frac{t}{\tau_{0}}}+B
$$

where, again, $A$ and $B$ are instrumental constants that can be directly obtained by measuring the signal values at $t=0$ and $t \gg 0$.

\section{Results}

We demonstrate our method on two $\mathrm{LiNbO}_{3}$ samples. The first one (cLN) is a typical sample with congruent composition ( $\mathrm{Li} / \mathrm{Nb}=48.6 \%$ ) with a length $L$ of about $30 \mathrm{~mm}$ along the beam propagation direction, which coincides with the $z$-axis. The applied voltage was in form of a square wave with peak to peak amplitude of $60 \mathrm{~V}$. As the thickness of the sample was about $4 \mathrm{~mm}$, this resulted in a field of about $15 \frac{\mathrm{kV}}{\mathrm{m}}$, which provided a sufficiently clear signal still remaining in the linear response range of the Tardy setup.

The second sample was a zinc-doped LN sample (Zn:LN) bulk doped by direct addition of $\mathrm{ZnO}$ to the melt. The $\mathrm{Zn}$ mole concentration in the bulk was determined to be $1.94 \mathrm{~mol}$. \%. The lenght along the propagation direction was about $L=5.4 \mathrm{~mm}$ while the thickness was $d=3.8 \mathrm{~mm}$. For this sample the square wave voltage had a peak-to-peak amplitude of $200 \mathrm{~V}$ corresponding to an applied field of about $52 \frac{\mathrm{kV}}{\mathrm{m}}$.

In Figure 2 it is reported a typical experimental result obtained in sample ( $\mathrm{Zn}: \mathrm{LN})$, in which the photodiode signal was properly converted to voltage units and noise filtered. The "bumps" visible in the oscilloscope trace are due to mechanical vibrations and power instabilities of our laser system. Each period of the curve could be satisfactorily fitted by Equation (6). It is important to stress that a similar fit quality could not be obtained with an exponential function and that doing so would have led to an overestimation of the characteristic time of a factor about 4.

For both samples, we repeated the measurement for different beam intensities. From the values of the characteristic time $\tau_{0}$ we obtained the photoconductivity as a function of the peak intensity, as reported in Figure 3. Both the samples display a linear dependence of the PC on the peak intensity, as expected and in agreement with previous literature. As the experimental points are characterized by different uncertainties we used a weighted linear regression to interpolate the two data sets. The data fits provide for the specific PC (i.e., PC normalized to the incident intensity) in the two samples the results reported in Table 1. The nonzero value of the intercept could be interpreted as dark conductivity, but this value appears to be much higher than published values [21]. However the errors are rather large so that this aspect needs to be better investigated in forthcoming experiments. 


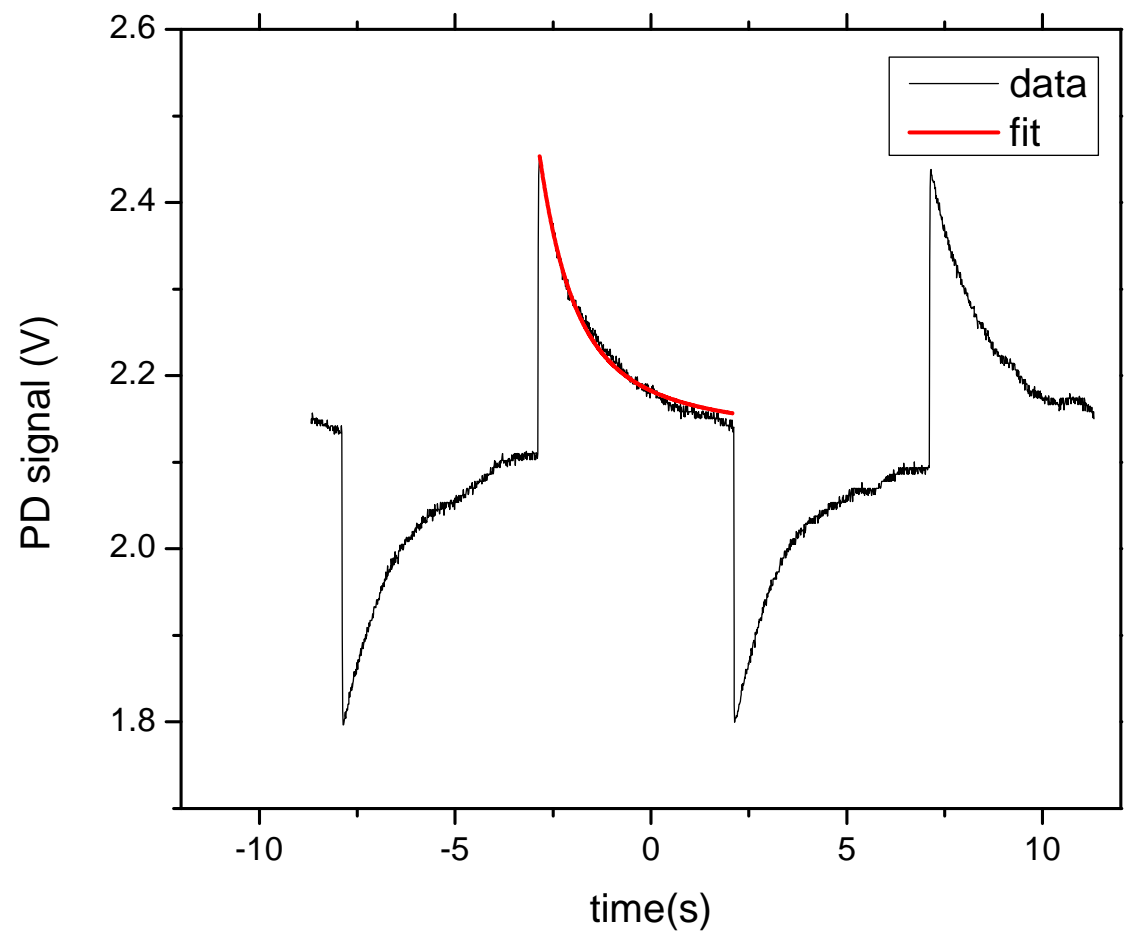

Figure 2. A typical experimental result obtained with our method. Sample Zn:LN, beam power $175 \mathrm{~mW}$. The red line is a fit to Equation (6).

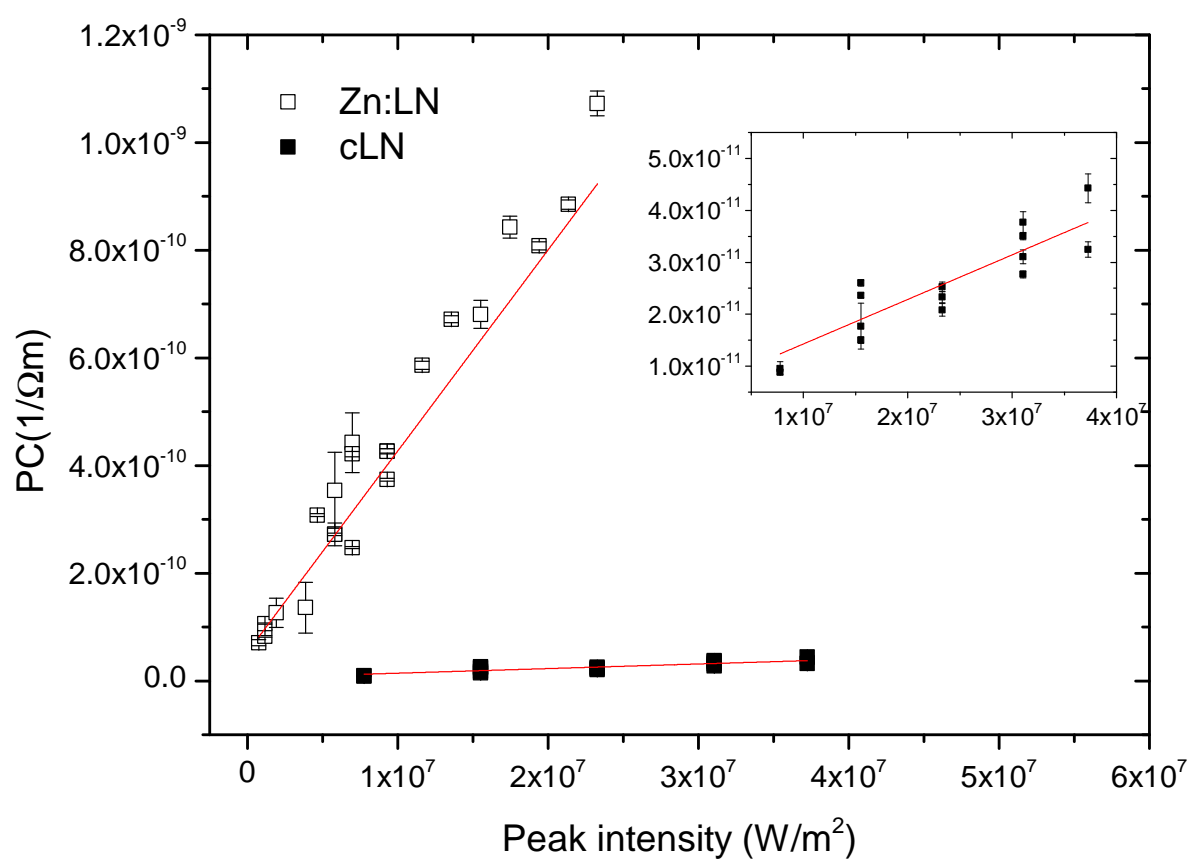

Figure 3. PC as a function of the peak beam intensity in sample cLN and Zn:LN. In the inset a zoomed view of data relative to sample cLN is shown for better clarity. The red lines are linear fits to the experimental data. 
Table 1. Specific PC values obtained in samples cLN and Zn:LN.

\begin{tabular}{|c|c|c|c|c|}
\hline Sample & $\begin{array}{c}\text { Specific PC } \\
\left(\times 10^{-19}(\Omega \mathrm{W})^{-1}\right)\end{array}$ & $\begin{array}{c}\text { Intercept } \\
\left(\times 10^{-12}(\Omega \mathrm{m})^{-1}\right)\end{array}$ & Ref. & Conditions \\
\hline cLN & $8.6 \pm 1.4$ & $5.7 \pm 2.8$ & This work & $\begin{array}{c}\text { Our method } \\
\lambda=514 \mathrm{~nm}, I_{\max }=4 \times 10^{7} \mathrm{~W} / \mathrm{m}^{2}\end{array}$ \\
\hline cLN & 20 & & [8] & $\begin{array}{c}\mathrm{I}-\mathrm{V} \text { measurements } \\
\lambda=532 \mathrm{~nm}, I_{\max }=10^{5} \mathrm{~W} / \mathrm{m}^{2}\end{array}$ \\
\hline Zn:LN 1.94 mol.\% & $370 \pm 20$ & $53 \pm 12$ & This work & $\begin{array}{c}\text { Our method; } \\
\lambda=514 \mathrm{~nm}, I_{\max }=4 \times 10^{7} \mathrm{~W} / \mathrm{m}^{2}\end{array}$ \\
\hline $\begin{array}{l}\text { Zn:LN } 2 \text { mol.\% } \\
\text { (nominal) }\end{array}$ & 1100 & & [22] & $\begin{array}{c}\text { Photoinduced birefringence; } \\
\lambda=514 \mathrm{~nm}, I_{\max }=1.2 \times 10^{7} \mathrm{~W} / \mathrm{m}^{2}\end{array}$ \\
\hline
\end{tabular}

As it can be expected on the basis of previous literature, the Zn:LN sample exhibits a specific PC about 40 times higher than the cLN sample, as expected in consequence of the elimination of $\mathrm{Nb}_{\mathrm{Li}}$ antisites which in congruent material are present in a large concentration (up to $\sim 19 \times 10^{25} \mathrm{~m}^{-3}$ ) and which can hang the motion of polaronic charges $[3,6]$. The order of magnitude of the observed values is in agreement with other measurements obtained with more elaborated methods $[3,22,23]$ and is reported in Table 1 . The differences with literature values may be ascribed to small differences in sample compositions or to the fact that in the cited papers the beam intensity is considered as uniform, while in our treatment we take into account also the gaussian intensity profile of our beam.

\section{Conclusions}

A simple method to measure the photoconductivity in LN is demonstrated. The method exploits the electro-optically induced changes as a probe of the electric field inside the sample, so that photoconductivity can be measured from the decay of the externally applied voltage. By choosing a specific experimental configuration ( $r_{22}$ configuration with circular input polarization) the influence of photorefractive phase changes can be minimized. Moreover the gaussian intensity profile of the illuminating beam has been taken into account obtaining a simple expression which can be used to fit the observed decays. Using this technique we were able to characterize the photoconductivity of two lithium niobate samples with different composition and different photoconductivity values. However, the approach is general and can be applied to a large class of electro-optic materials, provided that one can work out a configuration in which the measured refractive index changes can be attributed solely to the externally applied field and the influence of other parasitic phenomena (e.g., photorefractive or thermo-optic effects) can be neglected. In our realization of the method we used a Tardy's polarimetric setup to probe the time evolution of the internal field, but other approaches based e.g., on an interferometric reading could be used as well. The method provides reliable and repeatable measurements in a large range of photoconductivity values and can conveniently be adapted for in-situ measurements or high intensity studies in electro-optic and photorefractive materials.

Supplementary Materials: The Supplementary Materials are available at http:/ /www.mdpi.com/2073-4352/10/ 6/461/s1.

Author Contributions: Conceptualization, M.B. and L.G.; data curation, M.B. and L.G.; formal analysis, M.B. and L.G.; funding acquisition, M.A.; investigation, M.B., L.G. and M.A.; methodology, M.B. and L.G.; resources, M.A.; writing — original draft preparation, M.B.; writing — review and editing, M.A. and L.G. All authors have read and agreed to the published version of the manuscript.

Funding: This research received no external funding.

Conflicts of Interest: The authors declare no conflict of interest. 


\section{References}

1. Bazzan, M.; Sada, C. Optical waveguides in lithium niobate: Recent developments and applications. Appl. Phys. Rev. 2015, 2, 040603. [CrossRef]

2. Günter, P.; Huignard, J.P. (Eds.) Photorefractive Materials and Their Applications 1: Basic Effects; Springer: Berlin, Germany, 2006; Volume 113.

3. Volk, T.; Wöhlecke, M. Lithium Niobate; Springer-Verlag: Berlin/Heidelberg, Germany, 2009.

4. Guilbert, L.; Vittadello, L.; Bazzan, M.; Mhaouech, I.; Messerschmidt, S.; Imlau, M. The elusive role of $\mathrm{Nb}_{\mathrm{Li}}$ bound polaron energy in hopping charge transport in Fe:LiNbO 3 . J. Phys. Condens. Matter 2018, 30, 125701. [CrossRef] [PubMed]

5. Mhaouech, I.; Guilbert, L. Temperature dependence of small polaron population decays in iron-doped lithium niobate by Monte Carlo simulations. Solid State Sci. 2016, 60, 28-36. [CrossRef]

6. Vittadello, L.; Bazzan, M.; Messerschmidt, S.; Imlau, M. Small polaron hopping in Fe: $\mathrm{LiNbO}_{3}$ as a function of temperature and composition. Crystals 2018, 8, 294. [CrossRef]

7. Vittadello, L.; Bazzan, M.; Danielyan, A.; Kokanyan, E.; Guilbert, L.; Aillerie, M. A polaron approach to photorefractivity in Fe: $\mathrm{LiNbO}_{3}$. J. Phys. Commun. 2018, 2, 125003. [CrossRef]

8. Furukawa, Y.; Kitamura, K.; Takekawa, S.; Miyamoto, A.; Terao, M.; Suda, N. Photorefraction in $\mathrm{LiNbO}_{3}$ as a function of [Li]/[Nb] and MgO concentrations. Appl. Phys. Lett. 2000, 77, 2494-2496. [CrossRef]

9. Kostritskii, S.M.; Sevostyanov, O.G.; Aillerie, M.; Bourson, P. Suppression of photorefractive damage with aid of steady-state temperature gradient in nominally pure $\mathrm{LiNbO}_{3}$ crystals. J. Appl. Phys. 2008, 104, 114104. [CrossRef]

10. Arizmendi, L.; Powell, R. Anisotropic self-diffraction in Mg-doped LiNbO . J. Appl. Phys. 1987, 61, $2128-2131$. [CrossRef]

11. Günter, P.; Huignard, J.P. (Eds.) Photorefractive Materials and Their Applications 2: Materials; Springer: Berlin, Germany, 2007; Volume 114.

12. Bryan, D.; Gerson, R.; Tomaschke, H. Increased optical damage resistance in lithium niobate. Appl. Phys. Lett. 1984, 44, 847-849. [CrossRef]

13. Sommerfeldt, R.; Holtman, L.; Kraetzig, E.; Grabmaier, B. Influence of Mg doping and composition on the light-induced charge transport in $\mathrm{LiNbO}_{3}$. Phys. Status Solidi (A) 1988, 106, 89-98. [CrossRef]

14. Furukawa, Y.; Sato, M.; Bashaw, M.; Fejer, M.; Iyi, N.; Kitamura, K. Investigation of increased photorefractive damage resistance in $\mathrm{LiNbO}_{3}$ by two-wave mixing measurements. Jpn. J. Appl. Phys. 1996, 35, 2740-2744. [CrossRef]

15. Frejlich, J. Photorefractive Materials: Fundamental Concepts, Holographic Recording and Materials Characterization; Wiley: Hoboken, NJ, USA, 2006.

16. Zozulya, A.A.; Anderson, D.Z. Propagation of an optical beam in a photorefractive medium in the presence of a photogalvanic nonlinearity or an externally applied electric field. Phys. Rev. A 1995, 51, 1520-1531. [PubMed]

17. Fluck, D.; Brülisauer, S.; Günter, P. Photorefractive two-wave mixing with focused Gaussian beams. Opt. Commun. 1995, 115, 626-636. [CrossRef]

18. Jessop, H. On the Tardy and Sénarmont methods of measuring fractional relative retardations. Br. J. Appl. Phys. 1953, 4, 138. [CrossRef]

19. Weis, R.S.; Gaylord, T.K. Lithium niobate: Summary of physical properties and crystal structure. Appl. Phys. A 1985, 37, 191-203. [CrossRef]

20. Aillerie, M.; Theofanous, N.; Fontana, M.D. Measurement of the electro-optic coefficients: description and comparison of the experimental techniques. Appl. Phys. B 2000, 70, 317-334. [CrossRef]

21. Yevdokimov, S.; Yatsenko, A. Specific features of the dark conductivity in lithium niobate crystals of congruent composition. Phys. Solid State 2006, 48, 336-339. [CrossRef]

22. Aillerie, M.; Bourson, P.; Mostefa, M.; Abdi, F.; Fontana, M. Photorefractive damage in congruent $\mathrm{LiNbO}_{3}$. Part I. Zinc doped lithium niobate crystals. J. Phys. Conf. Ser. 2013, 416, 012001. [CrossRef]

23. Jermann, F.; Simon, M.; Krätzig, E. Photorefractive properties of congruent and stoichiometric lithium niobate at high light intensities. JOSA B 1995, 12, 2066-2070. [CrossRef]

(C) 2020 by the authors. Licensee MDPI, Basel, Switzerland. This article is an open access article distributed under the terms and conditions of the Creative Commons Attribution (CC BY) license (http://creativecommons.org/licenses/by/4.0/). 